\title{
DISTRIBUIÇÃO DE ELEMENTOS TÓXICOS NO ESTUÁRIO DO RIO AMAZONAS
}

Simone de Fátima Pinheiro PEREIRA ${ }^{\mathrm{a}}$

Otílio Othon PIRES ${ }^{\mathrm{a}}$

Augusto Fonseca SARAIVA ${ }^{\mathrm{b}}$

Geiso Rafael OLIVEIRA ${ }^{\mathrm{a}}$

Pedro Moreira de SOUSA JUNIOR ${ }^{\mathrm{a}}$

Rafaella Galvão MIRANDA ${ }^{\mathrm{a}}$

Cleber Silva e SILVA ${ }^{\mathrm{a}}$

Reginaldo da Silva SALES ${ }^{\text {a }}$

a - Laboratório de Química Analítica e Ambiental (LAQUANAM) - Instituto de Ciências Exatas e Naturais - Universidade Federal do Pará - Rua Augusto Correa, S/N - Guamá - Belém - PA Brasil, CEP 66075-900; Tel: 55-91-32018136; Fax: 55-91-32018136; Corresponding author: simonefp@ufpa.br

b - Laboratório Central - ELETRONORTE - Av. Tancredo Neves S/N - Terra Firme - Belém PA-Brasil

\section{Resumo:}

A presença de elementos não essenciais nas águas superficiais do rio Amazonas é uma preocupação global, o objetivo da pesquisa foi estudar a distribuição dos elementos As, Al, Mn, e $\mathrm{Pb}$ na água do estuário do rio Amazonas. As amostras foram coletadas em três regiões distintas: Canal Norte (AP), Canal Sul (PA) e rio Pará (PA) em três profundidades, com um total de 84 amostras. A espectrometria de emissão atômica com plasma indutivamente acoplado (ICPAES) foi utilizada para avaliar os teores de $\mathrm{Al}, \mathrm{Mn}$ e $\mathrm{Pb}$ e a espectrofotometria de absorção atômica com geração de hidretos (HGAAS) foi usada para a análise do As. O As variou de $<0,35$ 
a 50,60 $\mu \mathrm{g} / \mathrm{L}$, o Al de $<1,88$ a 3347,70 $\mu \mathrm{g} / \mathrm{L}$, o Mn de 0,13 a 403,39 $\mu \mathrm{g} / \mathrm{L}$ e $\mathrm{Pb}$ de $<0,18$ a 57,78 $\mu \mathrm{g} / \mathrm{L}$. Em média As (canal Sul), Al (todas as regiões), Mn (canal Norte e Sul) e Pb (canal Norte e Sul) se encontraram em valores acima do permitido pela legislação brasileira. Os elementos podem ter origem antrópica originária de atividade de mineração e industrial e origem natural proveniente de decomposição de rochas ígneas da bacia de drenagem do rio Amazonas que disponibilizam o elemento na forma ligada ao material particulado em suspensão.

Palavras-chave:Elementos tóxicos, foz do rio Amazonas, análise multivariada

\section{Introdução}

O rio Amazonas nasce a 5.300 metros de altitude na montanha Nevado Mismi, nos Andes peruanos. Já é reconhecido como o maior rio do mundo, tanto em extensão com $6.885 \mathrm{~km}$ como em volume d'água. A área de drenagem do rio Amazonas é de $6 \times 10^{6} \mathrm{~km}^{2}$ e sua descarga anual de $5,5 \times 10^{9} \mathrm{~m}^{3}$ ano ${ }^{-1}$, o equivalente a $1 / 5$ de todos os rios do planeta que representa cerca de $20 \%$ do fluxo mundial de água doce para o oceano [1].

A água é o principal veículo de transporte e distribuição de elementos químicos no ambiente. Geralmente os metais são transportados associados à partícula de sedimento e solo, ou de forma dissolvida nas águas dos rios, mares, estuários e na chuva [2]. Os elementos de baixo potencial iônico tendem a permanecerem em solução, os de potencial iônico intermediário geralmente apresentam pouca mobilidade geoquímica pela sua baixa solubilidade e forte tendência a adsorver nas superfícies de partículas finas de sedimentos [3].

A introdução dos elementos químicos para o meio onde ocorre sua dispersão depende do intemperismo químico, ou seja, da decomposição dos minerais das rochas, originadas por uma série de reações de oxidação, redução, hidrólise e hidratação. Esses processos se devem principalmente á ação das águas, ácido carbônico, ácidos fúlvicos, fúlmicos e chuva ácida [4].

Vários autores têm avaliado a presença dos elementos químicos na bacia do rio Amazonas [5], [6]. Seyler \& Boaventura [7] avaliaram a concentração e a variabilidade temporal de V, Cr, $\mathrm{Mn}, \mathrm{Co}, \mathrm{Cu}, \mathrm{Zn}, \mathrm{As}, \mathrm{Rb}, \mathrm{Sr}, \mathrm{Mo}, \mathrm{Cd}, \mathrm{Sb}, \mathrm{Cs}, \mathrm{Ba}, \mathrm{U}$ no rio Amazonas e seus afluentes principais (Negro, Madeira e Solimões) até a estação de Óbidos. Concluíram que As e Mn são elementos transportados por fluxos de inundação sugerindo uma lixiviação destes elementos na época da cheia de solos ricos em laterita que é uma formação que representa $80 \%$ do solo da bacia amazônica rica em Fe e Al. Miranda et al. [8] avaliaram a presença de $\mathrm{Ca}, \mathrm{Mg}, \mathrm{Al}, \mathrm{Fe}, \mathrm{Ba}, \mathrm{Mn}$, 
$\mathrm{Sr}, \mathrm{Ti}, \mathrm{Ag}, \mathrm{Be}, \mathrm{Cd}, \mathrm{Co}, \mathrm{Cr}, \mathrm{Cu}, \mathrm{Mo}, \mathrm{Ni}, \mathrm{Pb}$ e $\mathrm{Zn}$ na água do rio Tapajós no encontro com o rio Amazonas em frente a cidade de Santarém e encontraram os elementos Al e Fe em altas concentrações.

$\mathrm{O}$ objetivo deste estudo foi efetuar a caracterização dos elementos $\mathrm{As}, \mathrm{Al}, \mathrm{Mn}$ e $\mathrm{Pb}$ no estuário do rio Amazonas sua distribuição pelos canais Norte e Sul e rio Pará e estudar a significância dos resultados usando a estatística multivariada.

\section{Experimental}

As amostras foram coletadas na foz do rio Amazonas (figura 1) em triplicata da camada superficial $(1 \mathrm{~m})$, intermediária $(5 \mathrm{~m})$ e fundo $(10 \mathrm{~m})$ totalizando 84 amostras. As coletas foram realizadas com o auxilio de um navio da Marinha do Brasil (NP Bocaina) e lanchas de apoio ao longo do canal Norte (12 estações - N1 a N12), canal Sul (10 estações - S1 a S10) e rio Pará (6 estações - RP1 a RP6). Os locais de amostragem foram georeferenciados através de um GPS (global positioning system), modelo Garmin, Etrex. Para o planejamento da amostragem foi utilizada a tábua de maré fornecida pelo DHN e a carta náutica da área de estudo. A foz do Amazonas se constitui no grande depositário dos elementos químicos de toda a bacia do Amazonas daí sua importância como indicador do enriquecimento de metais na região. 


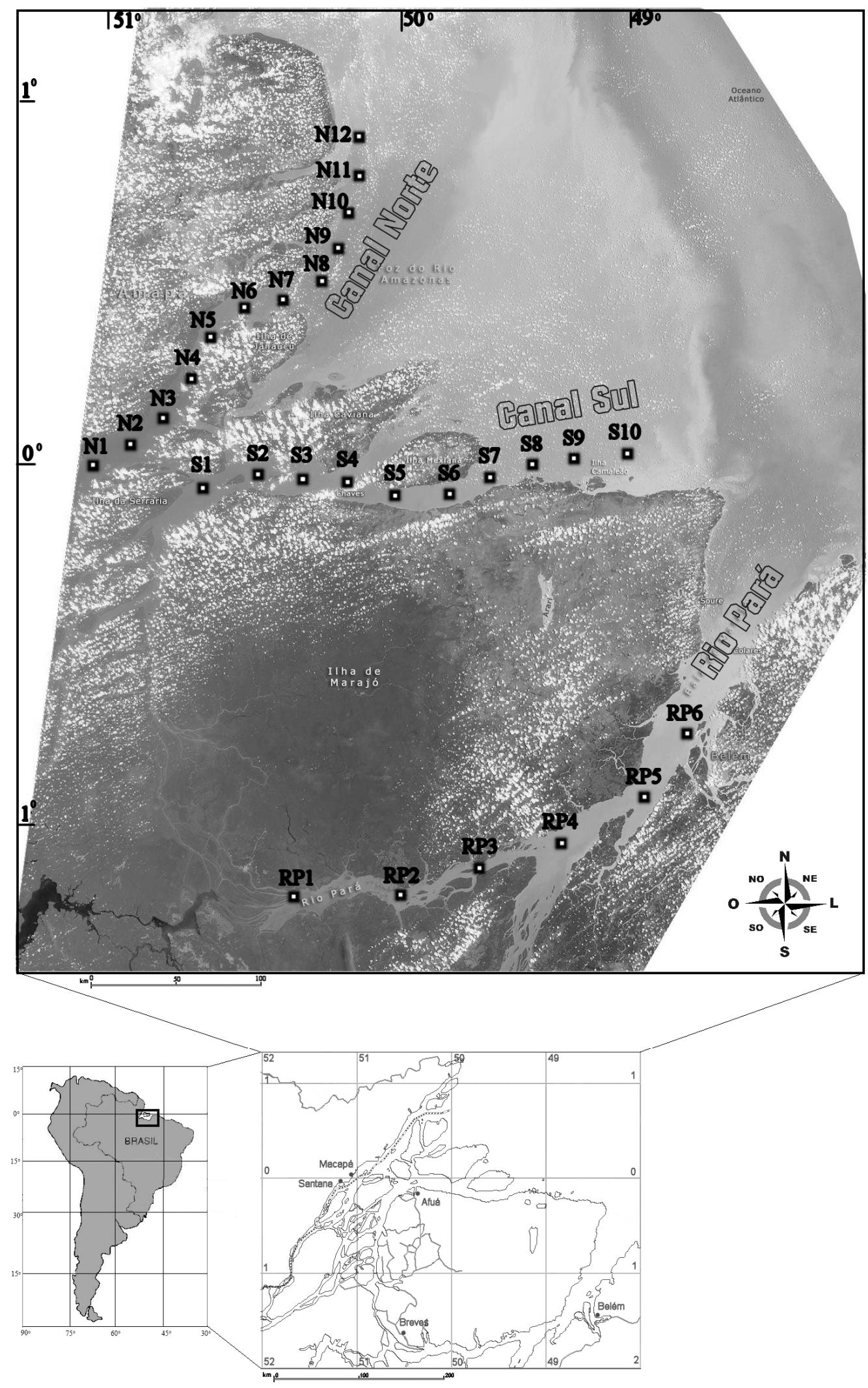

Ecl. Quím., São Paulo, 36 ,2011 49 
Figura 1. Foz do rio Amazonas com pontos amostrais

Fonte: Adaptado [9]

As amostras coletadas foram preservadas sob refrigeração a $4^{\circ} \mathrm{C}$ e encaminhadas ao laboratório para análise. Para a análise dos elementos solúveis as amostras foram filtradas em filtro milipore $0,22 \square \mathrm{m}$ que retêm pequenas partículas permitindo a análise somente dos elementos solúveis, acidificadas com ácido nítrico até pH 2 e encaminhadas para as análises.

Um espectrofotômetro de absorção atômica (AAS), modelo SPECTRAA-55, com acessório para geração de hidreto, modelo VGA-77, Varian Ind. \& Com. Ltda foi usado na análise do As e um espectrômetro de emissão atômica com plasma indutivamente acoplado (ICPAES), modelo Vista-Pro, Varian Ind. \& Com. Ltda foi usado na análise do Al, Mn e Pb.

A calibração dos instrumentos utilizados foi realizada através da confecção de curvas analíticas visando à determinação do coeficiente angular (a) e linear (b), e coeficiente de correlação (r) além do cálculo dos limites de detecção (LD) através da análise de 15 brancos (3S) e cálculo do limite de quantificação (LQ). Todas as curvas se mostraram lineares com coeficientes de correlação acima de 0,9945. Os resultados para o limite de detecção e limite de quantificação mostraram que a técnica do ICPAES pode ser usada na determinação de elementos traço em amostras de água, onde os mesmos se encontram em baixas concentrações. O estudo da exatidão foi realizado com a análise do material padrão de referência de água de rio (SRM 1640 NIST), no geral os resultados estão dentro da faixa aceitável para a recuperação dos padrões. Al apresentou excelente recuperação (96-101\%), Mn apresentou perdas (86-89\%) e As e Pb com incrementos na concentração (109-110\%).

Os resultados obtidos foram tratados utilizando-se estatística descritiva e multivariada como ferramenta auxiliar e os programas Microsoft ${ }^{\circledR}$ Office Excel 2007 e Statistica ${ }^{\circledR} 7$ no cálculo dos resultados. Os gráficos foram executados nos mesmos programas.

\section{Resultados e discussão}

Os resultados médios para a distribuição de $\mathrm{As}, \mathrm{Al}, \mathrm{Mn}$ e $\mathrm{Pb}$ na foz do rio Amazonas são apresentados na tabela 1, neste estudo o rio Amazonas foi classificado como rio de classe 2 de acordo com a resolução 357/05 do CONAMA. 
Tabela 1. Distribuição de As, Al, Mn e Pb no Canal Norte, Canal Sul e Rio Pará ( $\mu \mathrm{g} / \mathrm{L})$

\begin{tabular}{|c|c|c|c|c|c|c|c|c|c|c|c|c|c|c|}
\hline Estações & As & $\mathrm{Al}$ & $\mathrm{Mn}$ & $\mathrm{Pb}$ & Estações & As & $\overline{\mathrm{Al}}$ & $\mathrm{Mn}$ & $\mathrm{Pb}$ & Estações & As & $\overline{\mathrm{Al}}$ & $\mathrm{Mn}$ & $\mathrm{Pb}$ \\
\hline $\mathrm{RC} 357 / 05$ & 10 & 100 & 100 & 10 & RC357/05 & 10 & 100 & 100 & 10 & RC357/05 & 10 & 100 & 100 & 10 \\
\hline N1 & 3,10 & 472,26 & 57,75 & 28,67 & $\mathrm{~S} 1$ & 50,60 & 525,91 & 250,46 & $<0,18$ & RP1 & $<0,35$ & 106,74 & 41,81 & 13,44 \\
\hline $\mathrm{N} 2$ & 6,35 & $\mathbf{9 7 9 , 5 2}$ & 100,20 & 19,32 & $\mathrm{~S} 2$ & 8,33 & $\mathbf{1 7 5 , 8 2}$ & 253,37 & $<0,18$ & $\mathrm{RP} 2$ & 1,51 & 290,89 & 53,63 & $<0,18$ \\
\hline N3 & 3,38 & 3225,4 & 403,39 & 51,96 & S3 & 12,70 & 196,14 & 275,06 & 10,85 & RP3 & 6,84 & $<1,88$ & $<1,92$ & 12,23 \\
\hline N4 & 5,71 & 663,89 & 81,18 & $<0,18$ & S4 & 4,68 & 250,42 & 276,71 & 11,62 & RP4 & 7,03 & $<1,88$ & 17,19 & $<0,18$ \\
\hline N5 & 6,64 & 694,88 & 91,61 & $<0,18$ & S5 & 10,13 & 118,05 & 271,95 & $<0,18$ & RP5 & 4,77 & $<1,88$ & 8,18 & $<0,18$ \\
\hline N6 & 7,28 & 2295,50 & 184,05 & 41,72 & S6 & 4,70 & 60,54 & 238,04 & $<0,18$ & RP6 & 5,00 & 263,42 & $\begin{array}{l}108,0 \\
5\end{array}$ & 12,07 \\
\hline N7 & 7,81 & 2317,60 & 151,33 & 12,41 & S7 & 11,37 & 214,17 & 272,46 & $<0,18$ & & & & & \\
\hline N8 & 16,05 & 2302,90 & 167,83 & 14,59 & S8 & 7,72 & 257,13 & 266,51 & $<0,18$ & & & & & \\
\hline N9 & 7,58 & 3347,70 & 258,19 & 42,19 & S9 & 5,92 & 58,84 & 239,42 & $<0,18$ & & & & & \\
\hline N10 & 9,19 & 1686,80 & 129,98 & 9,80 & $\mathrm{~S} 10$ & 3,76 & 228,56 & 269,79 & $<0,18$ & & & & & \\
\hline N11 & 8,84 & 886,40 & 71,51 & $<0,18$ & & & & & & & & & & \\
\hline $\mathrm{N} 12$ & 14,22 & 978,08 & 85,67 & $<0,18$ & & & & & & & & & & \\
\hline Média & 8,01 & 1654,24 & 148,56 & 18,39 & Média & 11,99 & 208,56 & 261,38 & 2,25 & Média & 4,19 & 110,18 & 38,14 & 6,29 \\
\hline SD & 3,83 & 1018,45 & 98,72 & 18,64 & SD & 13,90 & 132,92 & 14,77 & 4,74 & SD & 2,86 & 136,07 & 39,83 & 6,91 \\
\hline Mínimo & 3,10 & 472,26 & 57,75 & $<0,18$ & Mínimo & 3,76 & 58,84 & 238,04 & $<0,18$ & Mínimo & $<0,35$ & $<1,88$ & $<1,92$ & $<0,18$ \\
\hline Máximo & 16,05 & 3347,70 & 403,39 & 51,96 & Máximo & 50,60 & 525,91 & 276,71 & 11,62 & Máximo & 7,03 & 290,89 & $\begin{array}{c}108,0 \\
5\end{array}$ & 13,44 \\
\hline
\end{tabular}

O As apresentou na foz do rio Amazonas uma média de 8,61 $\pm 8,98 \mu \mathrm{g} / \mathrm{L}$ variando de $<0,35$ a 50,60 $\mu \mathrm{g} / \mathrm{L}$. Constatou-se um enriquecimento do As de cerca de 4 vezes em relação ao encontrado por Seyler \& Boaventura [7], com alguns pontos (N8, N12, S1, S3, S5 e S7) apresentando teores acima do que estabelece a resolução 357/05 do CONAMA (10 $\mu \mathrm{g} / \mathrm{L})$, no 
canal Sul este enriquecimento é em torno de 7 vezes. O enriquecimento se repete no canal Norte e rio Pará em menor intensidade.

Os efeitos do As em excesso nos seres vivos envolvem danos em vários órgãos e sistemas como o sistema cardiovascular (distúrbios vasculares seguido de gangrena e doença do pé negro), sistema sangüíneo, sistema reprodutor (incremento de frequiência nos abortos espontâneos), pele (tumores, hiperpigmentação, hiperqueratose), fígado (disfunção hepática), sistema nervoso e sistema respiratório [10].

Förstner \& Wittmann [11] encontraram teores de As variando de 2,0-3,0 $\mu \mathrm{g} / \mathrm{L}$ em água de rio. O As de origem natural na foz do rio Amazonas pode ser explicado pelo transporte de materiais (dissolvidos, particulado), oriundos dos Andes, que atingem valores da ordem de 1,53 $\mu \mathrm{g} / \mathrm{L}$ de As dissolvido registrado no rio Solimões a $2.500 \mathrm{~km}$ da foz do rio Amazonas, estes valores vão diminuindo em direção a estação de Óbidos devido a diluição proveniente dos rios que drenam do escudo cristalino, que apresentam teores da ordem de $0,1 \mu \mathrm{g} / \mathrm{L}$ ou menores [7] e aumentam repentinamente na foz de acordo com os resultados obtidos neste estudo. A presença de As de origem antrópica na foz do rio Amazonas são explicados nos estudos de Seyler \& Boaventura [7] que realizaram estimativas sobre emissões atmosféricas de As oriundas de atividades antrópicas (notadamente desmatamentos e queimadas), que chegam à cerca de 250 toneladas de As anualmente. Outra possibilidade da origem antrópica do elemento na foz do Amazonas diz respeito a lixiviação de rejeitos ricos em As provenientes de uma usina de pelotização da mineradora ICOMI que atuava na cidade de Santana localizada as margens do rio Amazonas próxima a cidade de Macapá capital do estado do Amapá.

Segundo o box-plot da distribuição do As (figura 2) a maior variabilidade dos resultados foi observada no canal Sul que também apresentou os maiores resultados para o elemento. As condições de corrente e outros aspectos hidrológicos podem estar contribuindo para o enriquecimento do As neste local. Foram identificados dois resultado anômalos no canal Norte e um resultado extremo no canal Sul, entretanto, através do testes de hipóteses (teste de Dixon) foi observado que somente o resultado de As para a amostra S1 (Canal Sul) foi considerada fora da normalidade de resultados. 


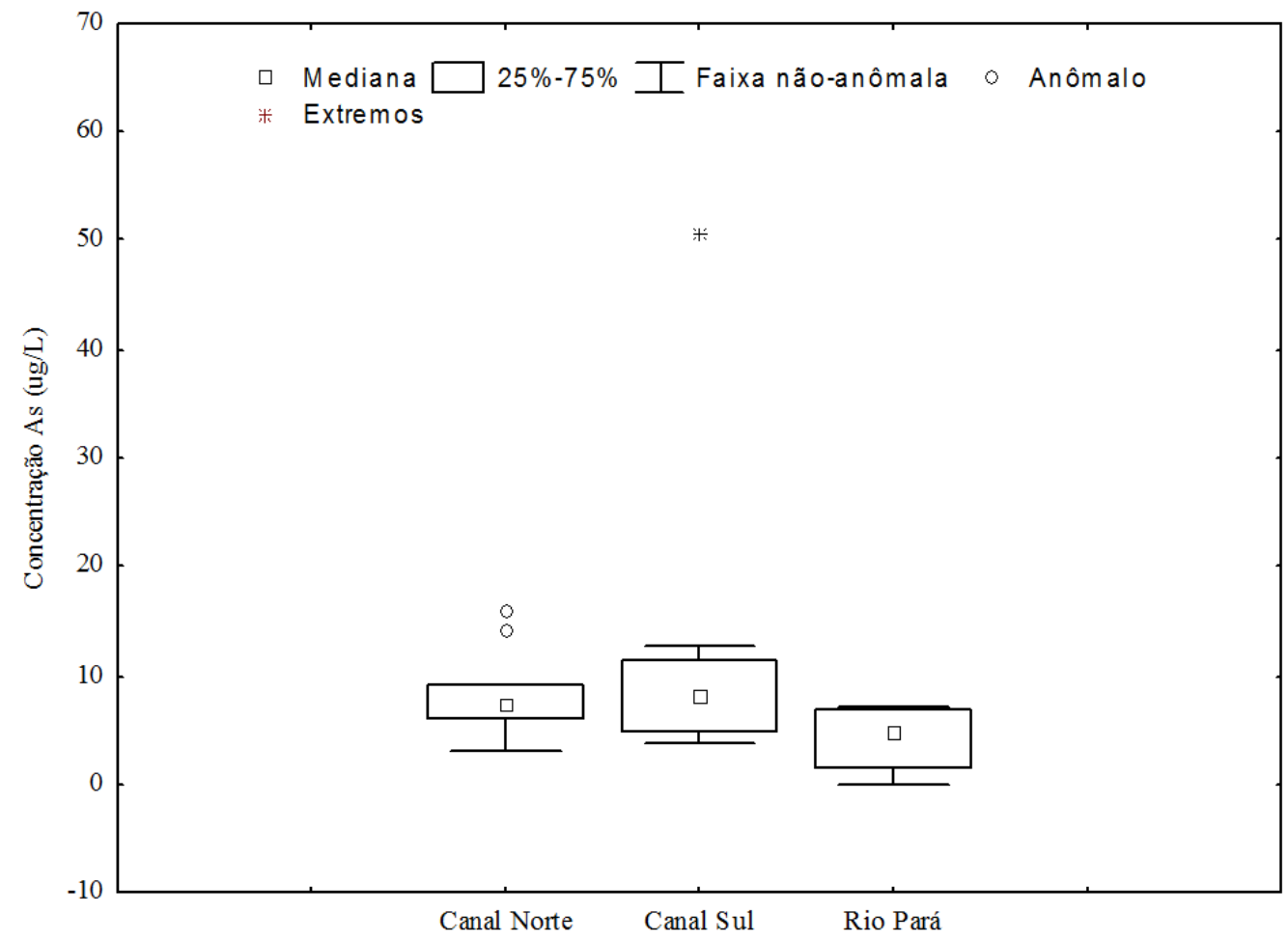

Figura 2. Box-plot da distribuição do As na foz do rio Amazonas

Nos estudos da correlação (Pearson) o As não apresentou correlações significativas com os outros elementos no canal Norte, apenas uma regular correlação negativa $(r=-0,428 ; \rho=0,1652$; y=35,0659 - 2,0814x) sem significância foi encontrada entre o As e Pb e no rio Pará a correlação negativa foi entre o As e $\mathrm{Al}(\mathrm{r}=-0,522 ; \rho=0,2882 ; \mathrm{y}=214,3588-24,855 \mathrm{x})$ também com pouca significância. A ausência de fortes correlações do As em relação aos outros elementos, principalmente $\mathrm{Al}$ e Mn no canal Norte e rio Pará mostra que nestes locais o elemento pode ter origem antrópica, já que o Al e Mn fazem parte da geoquímica local. No canal Sul foi encontrada uma excelente correlação positiva $(\mathrm{r}=0,833 ; \rho=0,0028 ; \mathrm{y}=113,0736+7,963 \mathrm{x})$ com um grau de confiança de $95 \%$ ou 0,05 entre o As e o Al indicando uma provável origem natural do As associada a intensa exploração da bauxita na região. Como as principais fontes de As natural são a arsenopirita e a pirita enriquecida em As, a exploração de depósitos contendo quantidades 
significativas destes minerais constitui também uma importante fonte natural de liberação de compostos de As para o ambiente [10].

Elevadas concentrações de Al com média de 807,06 $\pm 995,73 \mu \mathrm{g} / \mathrm{L}$, foram observadas para toda a região estuarina do rio Amazonas (variação de $<1,88$ a $3347,70 \mu \mathrm{g} / \mathrm{L}$ ), acima do que preconiza a resolução 357-05 do CONAMA (100 $\square \mathrm{g} / \mathrm{L})$ [12], estes altos valores podem estar associados a presença de argilominerais, principalmente a caulinita, tido como o principal contribuinte de Al na região amazônica. O Al ocorre no ambiente sob a forma de silicatos, óxidos e hidróxidos, combinado com outros elementos tais como sódio e flúor e complexado com a matéria orgânica. $\mathrm{O} A \mathrm{Al}$ é um constituinte maior e não reativo dos argilominerais, que por sua vez constituem a maior parte do material em suspensão e sedimentos [13]. O Al, embora seja o terceiro elemento mais abundante da crosta terrestre, possui reduzida função biológica. Esse elemento pode interferir no metabolismo de diversas formas de vida. No fitoplâncton, é capaz de inibir a absorção e os processos fisiológicos do fósforo; enquanto que, nos peixes, o excesso de Al torna-se uma potencial causa de problemas respiratórios. Existe forte possibilidade de que uma dieta contendo Al causa neurotoxicidade, principalmente a doença de Alzheimer [14].

De acordo com Baird [15] águas naturais apresentam concentração de Al na ordem de $26,98 \mu \mathrm{g} / \mathrm{L}$ valor em torno de 60 vezes menor que a média encontrada no canal Norte. Altas concentrações de Al também foram encontrados por Miranda et al. [8] que avaliaram o rio Tapajós na sua foz onde ocorre o encontro deste rio com o rio Amazonas, com teores médios de

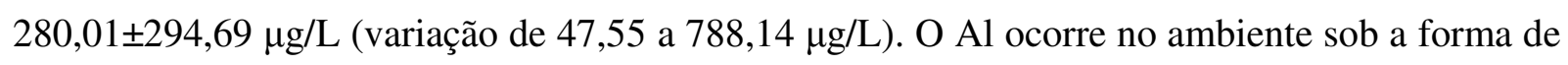
silicatos, óxidos e hidróxidos, combinado com outros elementos tais como sódio e flúor e complexado com a matéria orgânica. O Al é um constituinte maior e não reativo dos argilominerais, que por sua vez constituem a maior parte do material em suspensão e sedimentos. No ambiente estuarino o $\mathrm{Al}$ em solução é estabilizado em complexos orgânicos, podendo precipitar na forma de óxidos hidratados, e com o aumento do $\mathrm{pH}$ pode reagir com o silício e formar aluminossilicatos hidratados [13].

Pelo estudo do box plot (figura 3) percebe-se um incremento maior e uma maior variabilidade da concentração de Al no canal Norte, tendo sido verificado um resultado anômalo para o elemento no canal Sul, entretanto este valor não pode ser descartado de acordo com o teste Q. O Al se correlacionou positivamente com o Mn ( $\mathrm{r}=0,891 ; \rho=0,0001 ; \mathrm{y}=5,6682+0,0864 \mathrm{x})$ e $\mathrm{Pb}(\mathrm{r}=0,723 ; \rho=0,0079 ; \mathrm{y}=-3,4943+0,0132 \mathrm{x})$ no canal Norte, mesmo comportamento foi 
observado para a correlação do $\mathrm{Al}$ com o Mn no rio Pará $(\mathrm{r}=0,860 ; \rho=0,0279 ; \mathrm{y}=10,3987+$ $0,2518 x)$.

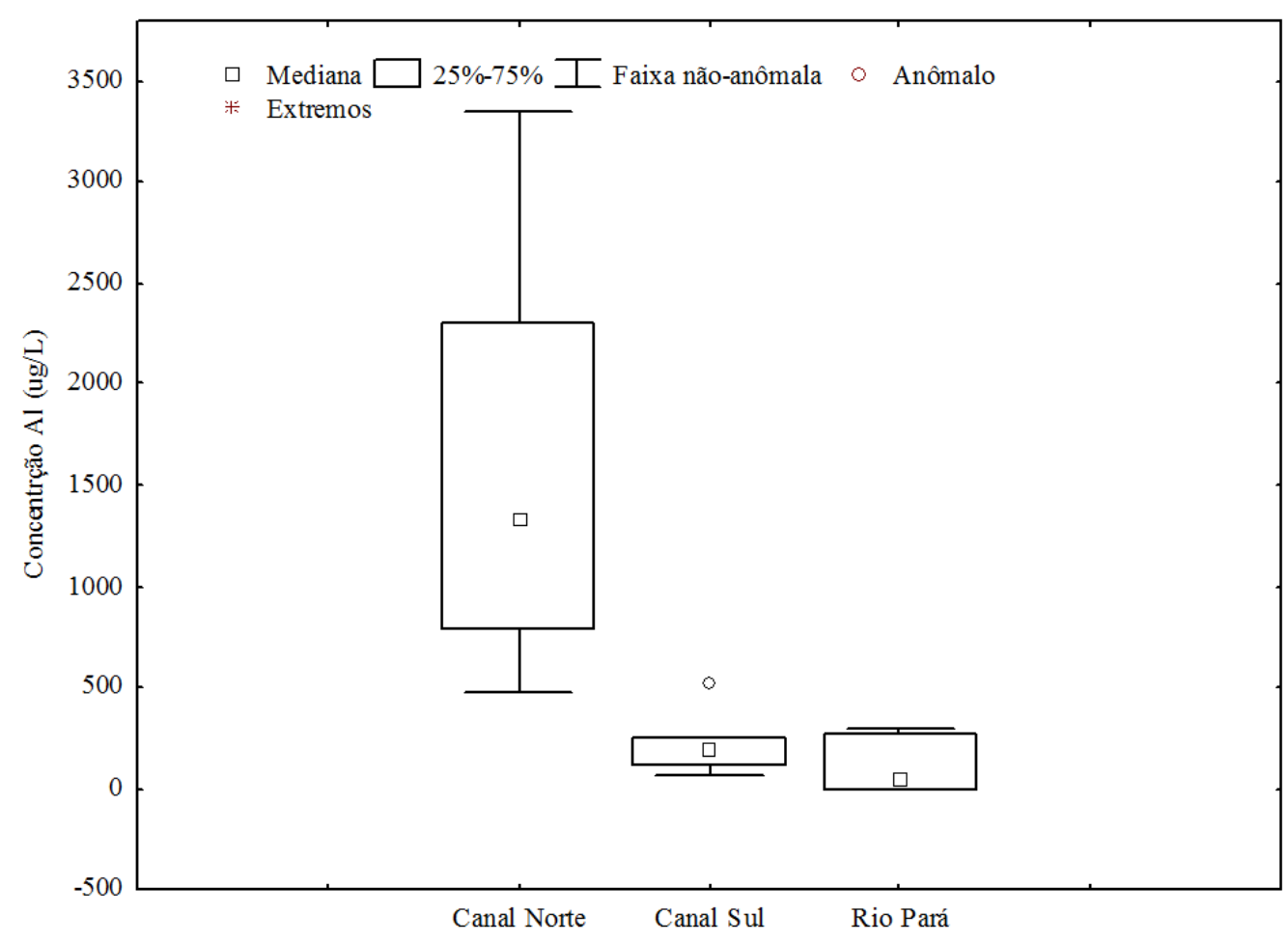

Figura 3. Box-plot da distribuição do Al na foz do rio Amazonas

O Mn está presente em águas naturais tanto na forma dissolvida ou em suspensão, que podem conter entre 1 e $500 \mu \mathrm{g} / \mathrm{L}$ de $\mathrm{Mn}$ total, podendo ser maior em áreas onde o $\mathrm{Mn}$ ocorre naturalmente [16]. O Mn variou de <1,92 a 403,39 $\square \mathrm{g} / \mathrm{L}$, com média de 165,19 $\pm 107,11 \square \mathrm{g} / \mathrm{L}$, na foz do rio Amazonas, teores acima da resolução 357/05 (100 $\square \mathrm{g} / \mathrm{L})$. O Mn foi encontrado em baixas concentrações por Miranda et al. [8] na foz do rio Tapajós, afluente do rio Amazonas, com teores médios de 12,02 $\pm 6,93 \mu \mathrm{g} / \mathrm{L}$ (variação de 2,20 a 22,95 $\mu \mathrm{g} / \mathrm{L}$ ). O Mn natural dissolvido é proveniente do intemperismo de seus compostos em rochas ígneas, sob forma de $\mathrm{Mn}^{2+}$, sendo precipitado com o aumento do $\mathrm{pH}$ [16]. O enriquecimento do $\mathrm{Mn}$ na foz do rio Amazonas pode ser devido à intensa exploração mineral de Mn ocorrida na área de Serra do Navio no Amapá por 50 anos e pela deposição de rejeitos ricos em Mn e em outros elementos oriundos de indústria de pelotização de minério na cidade de Santana. 
Segundo Salomons \& Forstner [17], este elemento pode ser liberado dos sedimentos recentemente depositados através de processos de difusão, bioturbação e erosão para ser novamente mobilizado. O Mn é amplamente distribuído na natureza, mas não ocorre como metal livre. Os compostos mais abundantes são os óxidos, sulfetos, carbonatos e os silicatos. A precipitação pode ocorrer sempre que o $\mathrm{pH}$ aumentar, desde que carbonatos ou silicatos presentes estejam em altas concentrações.

De acordo com o box-plot (figura 4) os teores de Mn no canal Norte apresentam maior variabilidade, os maiores valores foram observados no canal Sul com um valor anômalo no canal Norte que não foram descartados pelo teste $\mathrm{Q}$. $\mathrm{O} \mathrm{Mn}$ se correlacionou positivamente $\mathrm{com}$ o $\mathrm{Pb}$ no canal Norte $(\mathrm{r}=0,788 ; \rho=0,0023 ; \mathrm{y}=-3,7053+0,1487 \mathrm{x})$ e canal $\operatorname{Sul}(\mathrm{r}=0,518 ; \rho=0,1249 ; \mathrm{y}$ $=-41,2202+0,1663 \mathrm{x})$ com baixa significância.

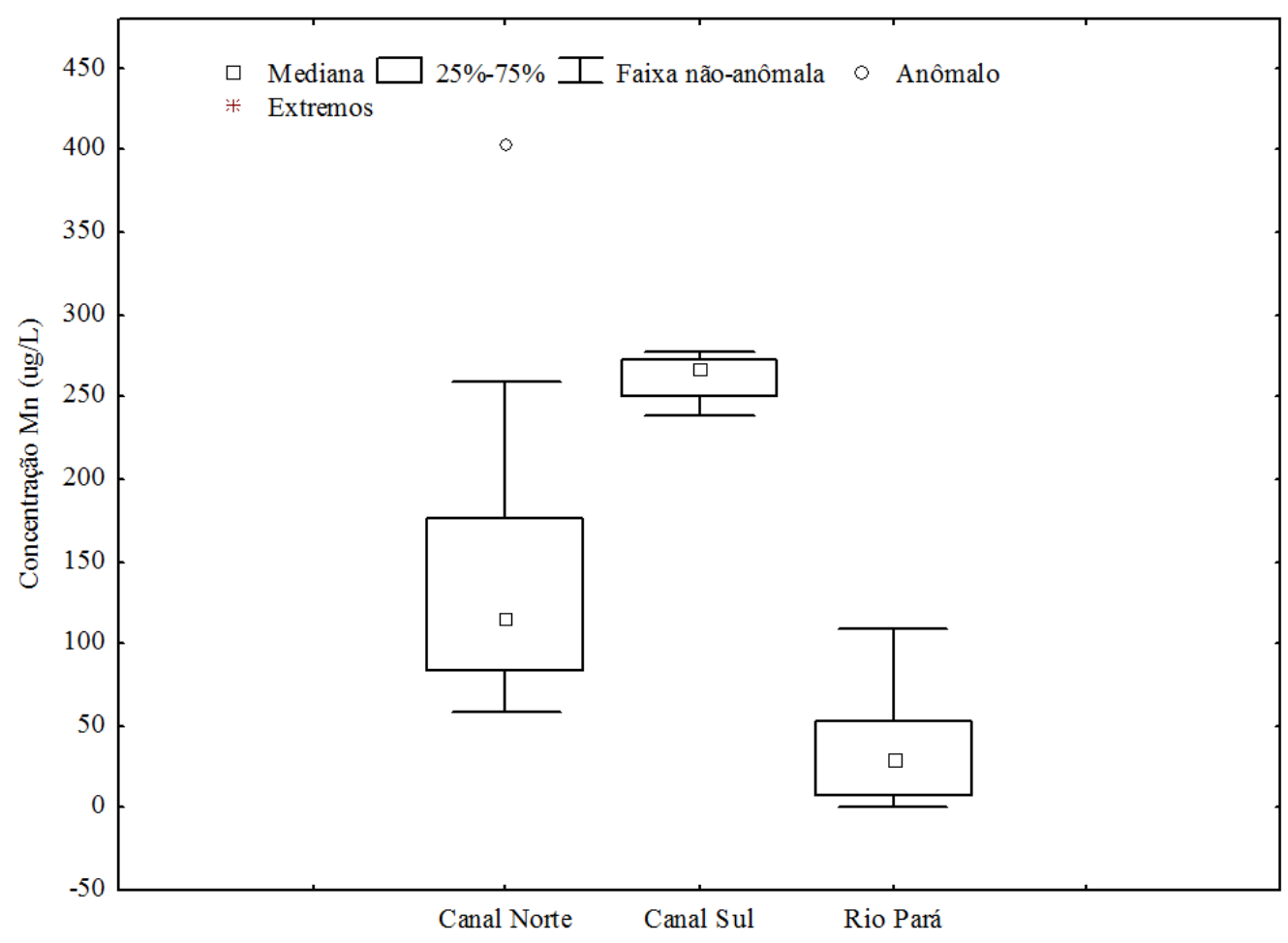

Figura 4. Box-plot da distribuição do Mn na foz do rio Amazonas

$\mathrm{O} \mathrm{Pb}$ se apresentou em altas concentrações com média de 10,03ะ14,64 $\mu \mathrm{g} / \mathrm{L}$ e variação de $<0,18$ a 51,96 $\mu \mathrm{g} / \mathrm{L}$, estes valores estão em não conformidade com a resolução 357/05 do 
CONAMA, indicando um enriquecimento, que pode ser de origem natural ou antrópica. Pereira et al. [18] avaliaram os níveis de $\mathrm{Pb}$ e outros elementos no rio Murucupi, e encontraram uma média de 2,8 $\pm 5,4 \mu \mathrm{g} / \mathrm{L}$ (variação de $<9,7$ a $15,0 \mu \mathrm{g} / \mathrm{L}$ ) para o $\mathrm{Pb}$, nível médio em torno de 4 vezes abaixo do encontrado neste estudo. O rio Murucupi é afluente do rio Pará e está localizado em área impactada pela indústria de $\mathrm{Al}$.

A intoxicação por $\mathrm{Pb}$ no homem causa danos ao sistema sangüíneo (destruição de glóbulos vermelhos), nervoso (cérebro, espinha dorsal, nervos sensores e motores), renal, cardiovascular [19]. O Pb que entra no organismo é inicialmente encontrado no sangue, eventualmente o $\mathrm{Pb}$ se deposita nos ossos, onde ele substitui o cálcio devido os íons $\mathrm{Pb}^{2+} \mathrm{e} \mathrm{Ca}^{2+}$ serem de tamanhos similares. $\mathrm{O} \mathrm{Pb}$ permanece no corpo humano por vários anos acumulando-se no organismo [15].

Livingstone [20] estimou que a média global do teor de $\mathrm{Pb}$ em lagos e rios é de 1-10 $\square \mathrm{g} / \mathrm{L}$. Embora essa estimativa inclua a contribuição antrópica, ela provavelmente ainda representa uma aproximação justa de condições naturais, pois a água que flui através dos ecossistemas tem uma considerável capacidade de auto-recuperação. Grandes descargas também podem ocorrer diretamente em águas naturais, mas, nesses casos, $\mathrm{o} \mathrm{Pb}$ tende a se localizar perto de pontos de descarga, devido à baixa solubilidade dos compostos que são formados em contato com o solo e a água [21]. Fontes de descargas de $\mathrm{Pb}$ na foz do rio Amazonas podem ter origem na exploração mineral e atividade industrial na área considerada. $\mathrm{O}$ maior teor de $\mathrm{Pb}$ foi encontrado na estação S1 (Canal Sul próximo a cidade de Santana (AP) e Afuá (PA)) local que apresentou também o maior teor de As. A presença de $\mathrm{Pb}$ e As em elevadas concentrações neste local necessita ser melhor avaliada em relação as prováveis fontes antrópicas de contribuição.

De acordo com o box-plot (figura 5) os maiores valores de $\mathrm{Pb}$ foram encontrados no Canal Norte, local com maior variabilidade de resultados, foi encontrado um resultado extremos para o $\mathrm{Pb}$ no canal Sul. 


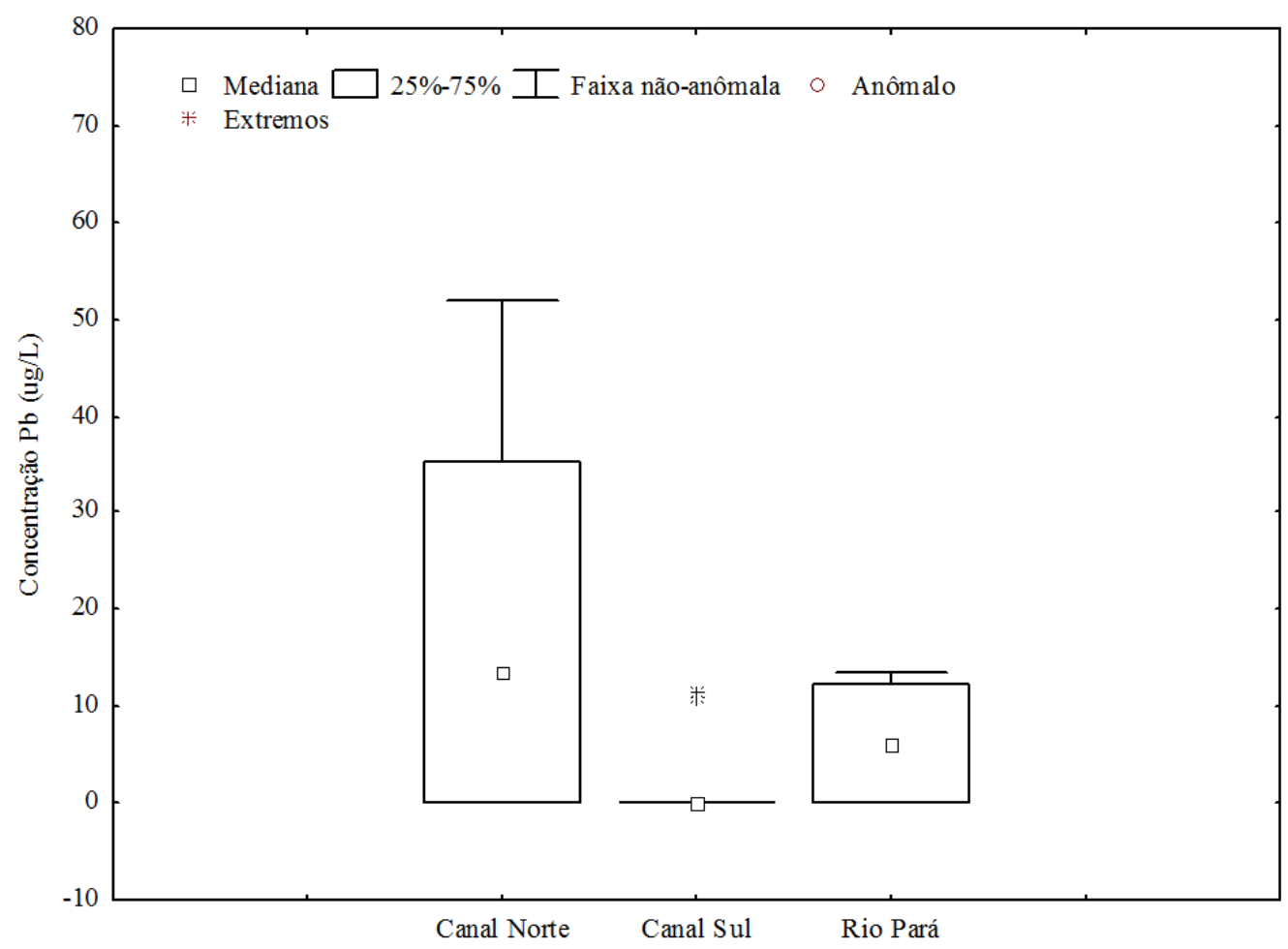

Figura 5. Box-plot da distribuição do $\mathrm{Pb}$ na foz do rio Amazonas

A maior contribuição dos elementos $\mathrm{As}, \mathrm{Al}, \mathrm{Mn}$ e $\mathrm{Pb}$ na foz do rio Amazonas dá-se apenas pelas três primeiras componentes principais, com seus respectivos valores de variância: componente $1(\mathrm{Al}=45,62 \%)$, componente $2(\mathrm{Mn}=38,23 \%)$ e a componente $3(\mathrm{~Pb}=12,32 \%)$, totalizando uma variância acumulada de 96,17\%. Com base nessas informações é possível afirmar que dentre as variáveis $\mathrm{As}, \mathrm{Al}, \mathrm{Mn}$ e $\mathrm{Pb}$ as que detêm a maior parte da informação $(96,17$ \%) sobre o conjunto de dados são: $\mathrm{Al}, \mathrm{Mn}$ e $\mathrm{Pb}$. O As se manteve isolado na análise de componentes principais (PCA) que comprova a provável origem antrópica do elemento.

Foi observado por meio do valor da significância (ANOVA) que as médias dos resultados das variáveis $\mathrm{Al}(\rho=3,08 \mathrm{E}-05), \mathrm{Mn}(\rho=5,23 \mathrm{E}-06)$ e $\mathrm{Pb}(\rho=0,022)$ diferem significativamente e são as que apresentam informações para explicar as características da área estudada, com um grau de confiança de $95 \%$ ou 0,05 . As médias para o As não diferiram significativamente com $\mathrm{F}$ calculado $<$ F crítico e significância de 0,239 .

No gráfico dos scores (figura 6) foi observada a formação de agrupamentos influenciados pelas variáveis selecionadas. O primeiro agrupamento é formado pelas amostras correspondentes ao canal sul (S) que são influenciadas pela concentração de Mn. O segundo agrupamento 
influenciado pelo $\mathrm{Al}$ e o $\mathrm{Pb}$ e constituído pelas amostras do canal norte $(\mathrm{N})$ e do rio Pará (RP) devido à característica de alguns metais que se encontram associados à fração dissolvida da água de apresentarem uma tendência pela forma iônica. Este fato foi evidenciado em trabalhos realizados por Cavalcante [22] na região sob influência da descarga do rio Amazonas.

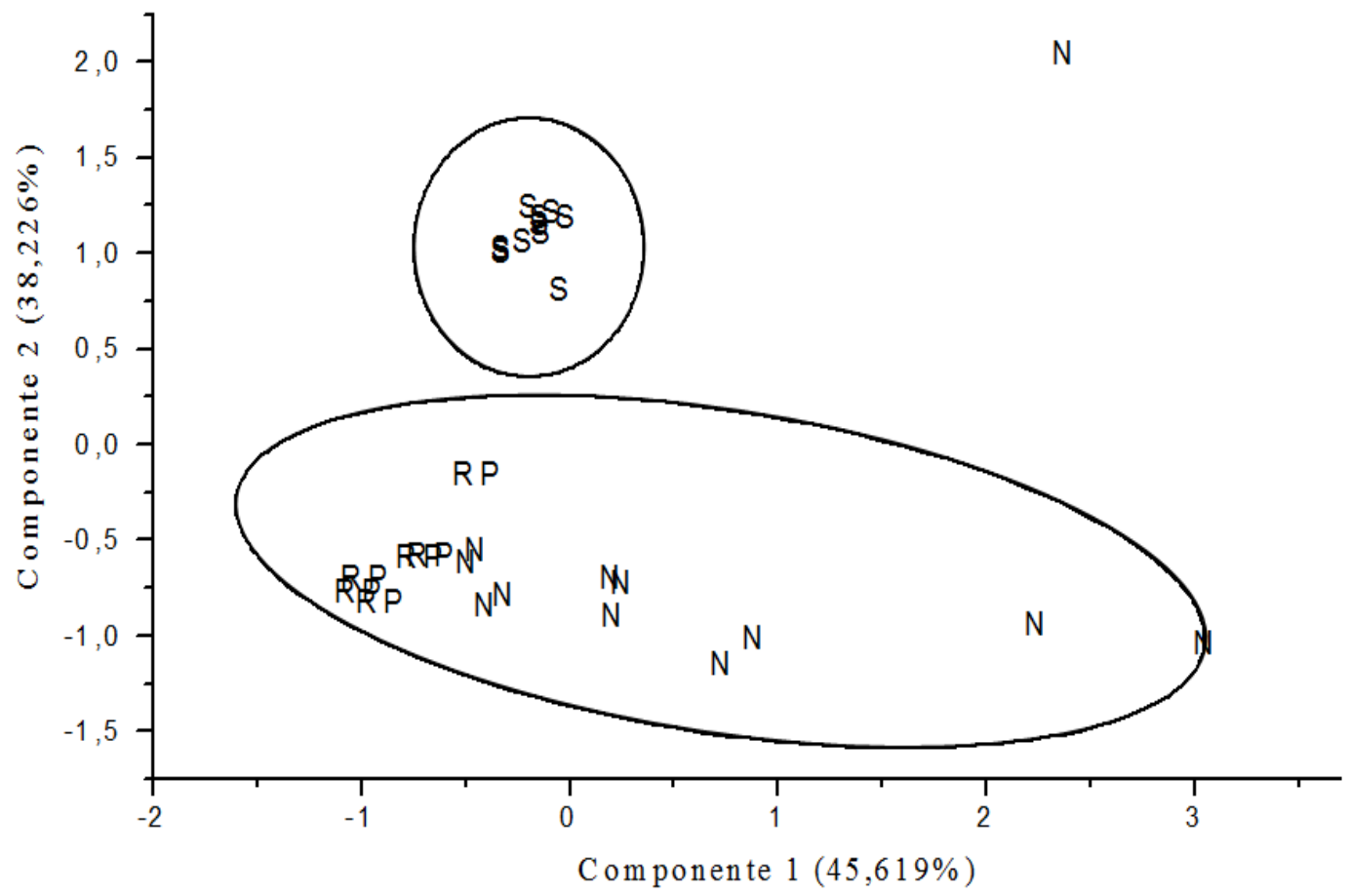

Figura 6. Gráfico dos scores destacando os agrupamentos de amostras.

O método de análise por agrupamento hierárquico (HCA) foi usado com o objetivo de verificar as semelhanças entre as amostras com base nos teores de elementos determinados para as diversas amostras na foz do rio Amazonas, através do cálculo de similaridade (calculada utilizando a distância euclidiana entre as amostras). $\mathrm{O}$ resultado de acordo com o dendrograma (figura 7) mostra a formação de três grupos, tomando-se como base uma distância euclidiana inferior a 500 unidades. 


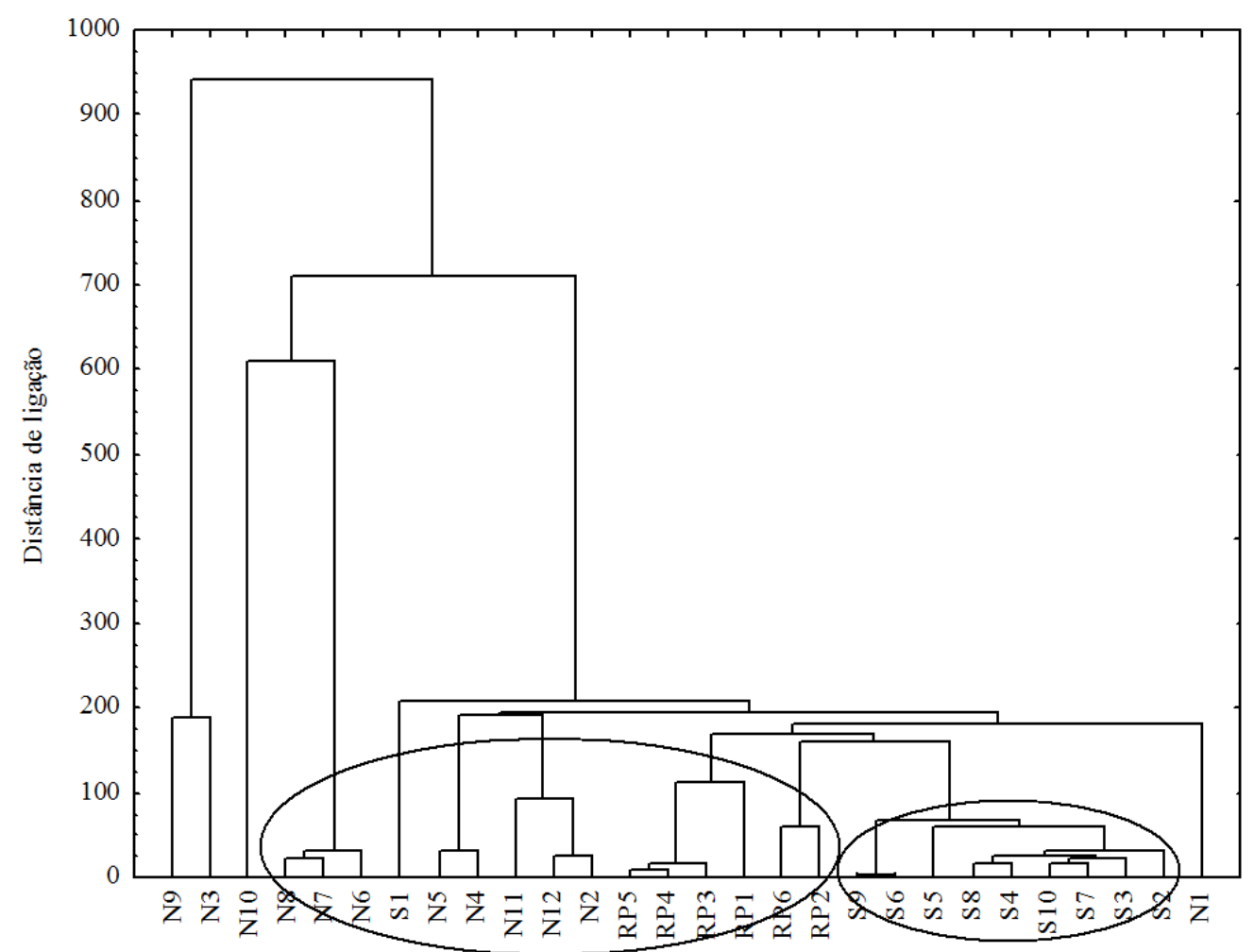

Figura 7. Dendrograma da distribuição de As, Al, Mn e Pb nos canais Norte e Sul e rio Pará

As principais similaridades observadas confirmam os resultados obtidos na análise de componentes principais, no que diz respeito à composição dos agrupamentos onde é observado um cluster que identifica uma grande similaridade entre os grupos das amostras correspondentes ao rio Pará (RP) e as amostras que formam o Canal Norte $(\mathrm{N})$ e os grupos das amostras do Canal Sul (S) separado dos demais indicando que esta área possui características únicas que contribuem para o enriquecimento anômalo dos elementos principalmente $\mathrm{As}$ e $\mathrm{Pb}$.

\section{Conclusões}

No presente estudo procurou-se mostrar a distribuição dos elementos $\mathrm{As}, \mathrm{Al}, \mathrm{Mn}$ e $\mathrm{Pb}$ no estuário do rio Amazonas e constatou-se a presença de elementos tóxicos, principalmente As e $\mathrm{Pb}$, com provável origem antrópica, os resultados deste estudo servem de alerta aos órgãos de controle ambiental, para um controle maior dos lançamentos de efluentes nos recursos hídricos locais. Os elementos se distribuíram pelas três áreas estudadas sendo mais evidente o enriquecimento dos elementos nos Canais Norte (Al, Mn) e Sul (As, Pb), o rio Pará sofre um enriquecimento próximo à área industrial de Barcarena-PA, local de industrialização de $\mathrm{Al}$ e outros produtos. Os teores médios para $\mathrm{Al}, \mathrm{Mn}$ e $\mathrm{Pb}$ estiveram em não conformidade com a 
resolução 357/05 do CONAMA, assumindo um caráter anômalo. O As apresentou elevadas concentrações principalmente no Canal Sul, próximo a cidade de Afuá na ilha do Marajó-PA. Similaridades foram encontradas entre os grupos de amostras oriundas do Canal Norte e Rio Pará comprovando a influência do Rio Amazonas na formação do Rio Pará. O Canal Sul ficou isolado dos demais indicando a influência do Oceano Atlântico e de outros fatores hidrodinâmicos e geoquímicos característicos da área da Ilha do Marajó no enriquecimento dos elementos tóxicos.

\section{Agradecimentos}

Agradecemos o apoio da Eletronorte, CNPq, CAPES, FINEP e ao Programa de Pós-Graduação em Química da UFPA.

PEREIRA, S.F.P.; PIRES, O.O.; SARAIVA, A.F.; OLIVEIRA, G.R.; SOUSA JUNIOR, P.M.; MIRANDA, R.G.; SILVA, C.S.; REGINALDO, S.S.

\section{TOXIC ELEMENTS DISTRIBUTION IN THE AMAZON RIVER ESTUARY}

Abstract: The non-essential elements found in the superficial waters of the Amazon River is a global worry since the local hydric resources represent the world's largest fresh water reservoir. This research aimed at studying the occurrence of $\mathrm{As}, \mathrm{Al}, \mathrm{Mn}$ and $\mathrm{Pb}$ in the waters of the Amazon River estuary. The 84 samples in three depths were collected in three different regions: North Channel of the Amazon River, in the state of Amapá; South Channel of the Amazon River, in the state of Pará; and Pará River, also in the state of Pará. Inductively coupled plasma atomic emission spectrometry (ICPAES) method was employed to determine the concentrations of $\mathrm{Al}$, $\mathrm{Mn}$ and $\mathrm{Pb}$ in the samples while hydride generation atomic absorption spectrometry (HGAAS) method was employed in determining their As concentrations. As concentrations varied from $<0.35$ to $50.60 \mu \mathrm{g} / \mathrm{L} ; \mathrm{Al}$ concentrations varied from $<1.88$ to $3347.70 \mu \mathrm{g} / \mathrm{L}$; Mn concentrations varied from $<1.92$ to $403.39 \mu \mathrm{g} / \mathrm{L}$; and $\mathrm{Pb}$ concentrations varied from $<0.18$ to $51.96 \mu \mathrm{g} / \mathrm{L}$. On average, the concentrations of As (samples from the South Channel), of Al (samples from the three regions), of $\mathrm{Mn}$ (samples from the North Channel and from the South Channel), and of $\mathrm{Pb}$ (samples from the North Channel) were higher than allowed by the Brazilian legislation. The origin of the elements can be anthropogenic due to mining and industrial activities as well as natural due to decomposition of igneous rocks from the drainage basin of the Amazon River which releases the element linked to the suspended particulate matter. High concentrations of toxic elements, especially of $\mathrm{As}$ and $\mathrm{Pb}$, put the health of the populations who consume water of the Amazon River and the Pará River at risk. 
Keywords: Toxic elements, Amazon River mouth, multivariate study

\section{Referências}

[1] M. Molinier, J.L. Guyot, J. Callède, V. Guimarães, E. Oliveira, N. Filizola, Hydrologie du bassin amazonien, Environnement et développement en Amazonie brésilienne, Théry H. (ed.), Publ. Belin, Paris, 1997, 24.

[2] R. Cidu, R. Biddau, Applied Geochemistry. 22(12) (2007) 2777.

[3] J.C. Fernandez-Caliani, C. Barba-Brioso, J.D. de la Rosa, Geoderma. 149(3-4) (2009) 393.

[4] M.L.W. Tuttle, G.N. Breit, M.B. Goldhaber, Applied Geochemistry. 24(8) (2009) 1565.

[5] J. Viers, G. Barroux, M. Pinelli, P. Seyler, P. Oliva, B. Dupre, G.R. Boaventura, Science of the Total Environment. 339(1-3) (2005) 219.

[6] A.-M. Aucour, F.-X. Tao, P. Moreira-Turcq, P. Seyler, S. Sheppard, M. F. Benedetti, Chemical Geology. 197(1-4) (2003) 271.

[7] P.T. Seyler, G.R. Boaventura, Trace elements in the mainstream Amazon River. In: M.E.

McClain, F.R.L.Victoria, J.E. Richey, (Eds.) The Biogeochemistry of the Amazon Basin. Oxford Press, (2001) 307.

[8] R.G. Miranda, S.F.P. Pereira, G.R. Oliveira, D.T.V. Alves, Revista Ambiente \& Água. 4 (2009) 75 .

[9] Photo of Amazon River's Mouth, Brazil, September. Acesso em 10/12/09, Disponível em: http://www.zonu.com/brazil_maps (2000).

[10] WHO - World Health Organization, Arsenic and Arsenic Compounds. Environmental Health Criteria. Geneva, (2001) 224.

[11] U. Förstner, G.T.W. Wittmamn, Metal Pollution in the Aquatic. Environment. Berlin, Springer-Verlag. (1983) 486.

[12] CONAMA. Conselho Nacional do Meio Ambiente, Resolução no 357/05. Acesso em 07/08/09, Disponível em: http://www.mma.gov.br/port/conama/res/res05/ res35705.pdf, (2005).

[13] E.R. Sholkovitiz, N.B. Price, The major element of suspended matter in Amazon estuary. Geochimica Cosmochimica Acta. 44(2) (1980) 163.

[14] World Health Organization, Aluminium. Environmental Health Criteria, Geneva, (1997) 194.

[15] C. Baird, Environmental Chemistry. $2^{a}$. ed., W. H. Freeman and Company, New York, (1999) 557. 
[16] World Health Organization, Manganese. Environmental Health Criteria, Geneva, (1981) 17.

[17] W. Salomons, U. Förstner, Metals in Hidrocycle. Berlin, Springer-Verlag, (1984) 349.

[18] S.F.P. Pereira, M.A. Lima, K.H. Freitas, C.S. Mescouto, A.F. Saraiva, Revista Ambiente \& Água. 2(3) (2007) 62.

[19] L. W. Newland, K. A. Daum, Lead. In: Hutzinger, O. The Handbook of Environmental Geochemistry. 3, part B. Anthropogenic Compounds. Berlin, Springer-Verlag: (1982) 1.

[20] D.A. Livingstone, Data of geochemistry. Sixth Edition. Chapter of chemical composition of rivers and lakes. US Geol. Survey Prof. Paper (1963) 440.

[21] World Health Organization, Lead. Environmental Health Criteria, Geneva, (1977) 3.

[22] P.R.S. Cavalcante, Partição de metais de transição na foz do rio Amazonas. Universidade Federal Fluminense. Rio de Janeiro. Dissertação de Mestrado em Geoquímica, (1991) 127. 